\title{
Generation of ramp waves using variable areal density flyers
}

\author{
R. E. Winter - M. Cotton - E. J. Harris • \\ D. J. Chapman · D. Eakins
}

Received: 30 July 2014 / Revised: 13 November 2014 / Accepted: 31 January 2015 / Published online: 19 February 2015

(C) The Author(s) 2015. This article is published with open access at Springerlink.com

\begin{abstract}
Ramp loading using graded density impactors as flyers in gas-gun-driven plate impact experiments can yield new and useful information about the equation of state and the strength properties of the loaded material. Selective Laser Melting, an additive manufacturing technique, was used to manufacture a graded density flyer, termed the "bed-of-nails" (BON). A 2.5-mm-thick $\times$ 99.4-mm-diameter solid disc of stainless steel formed a base for an array of tapered spikes of length $5.5 \mathrm{~mm}$ and spaced $1 \mathrm{~mm}$ apart. The two experiments to test the concept were performed at impact velocities of 900 and $1100 \mathrm{~m} / \mathrm{s}$ using the $100-\mathrm{mm}$ gas gun at the Institute of Shock Physics at Imperial College London. In each experiment, a BON flyer was impacted onto a copper buffer plate which helped to smooth out perturbations in the wave profile. The ramp delivered to the copper buffer was in turn transmitted to three tantalum targets of thicknesses 3,5 and $7 \mathrm{~mm}$, which were mounted in contact with the back face of the copper. Heterodyne velocimetry (Het-V) was used to measure the velocity-time history, at the back faces of the tantalum discs. The wave profiles display a smooth increase in velocity over a period of $\sim 2.5 \mu \mathrm{s}$, with no indication of a shock jump. The measured profiles have been analysed to generate a stress vs. volume curve for tantalum. The results have been compared with the predictions of the Sandia National Laboratories hydrocode, CTH.
\end{abstract}

Communicated by W. Proud.

R. E. Winter $(\bowtie) \cdot$ M. Cotton · E. J. Harris

AWE, Aldermaston, Reading, UK

e-mail: rwinter1@imperial.ac.uk

D. J. Chapman · D. Eakins

Institute of Shock Physics, Imperial College, London, UK
Keywords Selective laser melting - Additive manufacture . Ramp wave · Impact

\section{Introduction}

It is well known that experiments to measure the changing profiles of ramp compression waves can provide useful data on the material through which the wave propagates (for example, see $[1,2])$. One way of generating a ramp wave is to impact a disc of the specimen material with a flyer whose density is low at the leading face but increases with distance from that face. In this paper, we explore the potential of a flyer, manufactured by selective laser melting (SLM), such that the average (or areal) density varies in a way which generates ramp waves in an impacted target. The procedure was as follows. A stainless steel flyer was designed in which the areal density varied over an axial distance of $5.5 \mathrm{~mm}$. The region of varying density was built on a 2.5 -mm-thick solid base (also manufactured by SLM). The Sandia National Laboratories code CTH was used to compute the wave delivered to a series of solid targets at a range of impact velocities. Based on the computational study, experiments were designed with the objective of demonstrating the bed-of-nails concept for generating ramp waves and determining the validity of CTH to compute the configuration. Experiments were performed and the results were compared with CTH. A simple analysis was used to illustrate how longitudinal stress vs. specific volume relationships could be constructed for the target material.

\section{The "bed-of-nails" concept}

A bed-of-nails (BON) flyer was manufactured by SLM. In the procedure used in this work, a $\sim 40 \mu \mathrm{m}$ layer of $316 \mathrm{~L}$ 
Fig. 1 a The "bed-of-nails" array. The nails merge with a 2.5-mm-thick disc and are bordered by a 0.5 -mm-thick steel rim. The parabolic profile gives a linear density variation through the thickness of the disc. b The cut-down variant used in some simulations. c Nail profiles (see main text for further details)

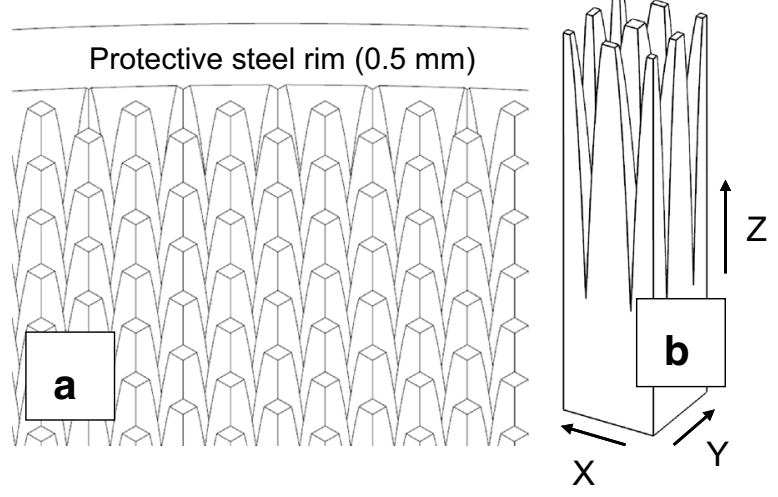

stainless steel powder was deposited on a base plate. A laser beam with a diameter of $\sim 40 \mu \mathrm{m}$ was scanned over the layer,

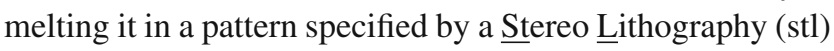
file. An stl file is a format used to export a model from a Computer Aided Design (CAD) package. The structure was built up by adding more layers. The density variation was achieved by building a "bed-of-nails" structure on a $2.5-\mathrm{mm}$ thick base plate. Our initial target was to fabricate a structure in which the density varied linearly with distance from the impact surface. We judged that the simplest way to do this was to construct individual nails whose cross-sectional area forms a square with an area that increased linearly with distance from the impact face. In principle, this aim can be achieved by assigning a parabolic profile to the nail with the tips of the nails co-incident with the leading face of the flyer. However, in view of the limited spatial resolution of the SLM build process, the tips of the needles were trimmed as illustrated in Fig. 1. The length of the trimmed nails was $5.5 \mathrm{~mm}$. They were centered on a $1 \mathrm{~mm} \times 1 \mathrm{~mm}$ grid and sat on a $2.5-\mathrm{mm}$ thick base of solid stainless steel. The form of the nails used in the initial CTH assessment is shown by the two solid black lines in Fig. 1c. The profile indicted by the two dashed lines will be discussed later. Our coordinate system is defined in Fig. 1b.

\section{CTH simulations}

Confidence in the ability of the BON concept to generate useful ramp waves was gained by running CTH simulations. CTH is a multi-dimensional, multi-material Eulerianbased code developed by Sandia National Laboratories [3]. It is suitable for the simulation of large deformations and material distortions loaded under shock and high strain rate conditions. CTH incorporates many popular equationsof-state (e.g. Mie-Gruneisen) and strength models (e.g. Steinberg-Guinan [4], Johnson-Cook [5]) used to describe the behaviour of materials subjected to high-rate loading. It has a 3D capability together with the ability to read in descriptions of shapes defined using the stereolithography representation (stl) generated with computer aided design (CAD) software. In previous work, we have studied the response of cellular materials manufactured using SLM to shocks delivered by projectiles in the velocity regime 300 $900 \mathrm{~m} / \mathrm{s}$ [6]. The results compared well with CTH predictions giving confidence in the code in a regime similar to that envisaged for the ramp wave studies described in this paper.

A series of simulations based on the BON concept were run using $\mathrm{CTH}$ with the aim of estimating the rate of pressure rise and the smoothness of the wave delivered to a range of target materials. The constitutive model used for the stainless steel was described in [6]. Initial simulations were based on the expected experimental flyer which measured $99.4 \mathrm{~mm}$ diameter $\times 8 \mathrm{~mm}$ thick. However, later in the study, computer time was saved by reducing the configuration to a $2 \mathrm{~mm} \times 2 \mathrm{~mm}$ element with reflective boundaries as shown in Fig. 1b. To test for mesh convergence, one variant of the reduced configuration was run with both 50 and $25 \mu \mathrm{m}$ meshes. The smaller mesh gave slightly more detailed definition in the images of the material distortion in the vicinity of the tips of the impacting nails but had no noticeable effect on the stress patterns delivered to the target. From this evidence, we concluded that the simulations could be considered mesh converged at $50 \mu \mathrm{m}$. Therefore, all future full and reduced configurations were run with $50 \mu \mathrm{m}$ meshing.

The first assessment of the wave delivered by the BON flyer was made by running CTH simulations in which a 16mm-long copper target was impacted by a $99.4-\mathrm{mm}$-diameter BON flyer at $900 \mathrm{~m} / \mathrm{s}$. The choice of a relatively thick target ensured that complications due to the reflection of the impact shock from the rear surface of the target would have minimal effect. Further, it was envisaged that using a material whose shock properties are relatively well known would give confidence that the simulations would be reasonably representative of reality. Initially, for simplicity, the copper was treated as a fluid (no strength). 


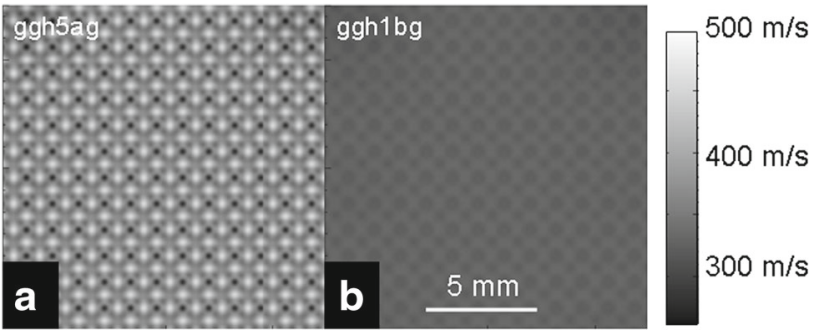

Fig. 2 Particle velocity distributions on the $x-y$ plane in material which was initially $3 \mathrm{~mm}$ from the impact face for the simulation depicted in Fig. 3. a and $\mathbf{b}$ Show the pressure distributions at $1.2 \mu$ s from impact time predicted by CTH when a the copper is treated as a fluid and $\mathbf{b}$ a Johnson-Cook strength model is assigned to the copper. The alphanumerical combinations in the upper left corner of each image identify the run number of the simulation

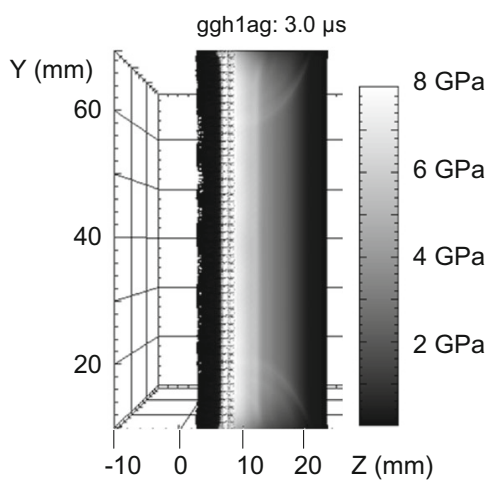

Fig. 3 Cross section showing the stress distribution following a 900 $\mathrm{m} / \mathrm{s}$ impact of the BON flyer onto a 16-mm copper target at $3.0 \mu$ s from impact. At the instant of impact, the flyer extends from 0 to $8 \mathrm{~mm}$ and the copper target extends from 8 to $22 \mathrm{~mm}$

The simulations suggested that the pressure profile generated in the target using this model would be unacceptably perturbed in both the direction of wave propagation $(z)$ and in the plane of the wave front ( $x-y$ plane). For example, Fig. 2a is a snapshot of the $x-y$ plane at $1.2 \mu$ s obtained with a fluid copper model. The plot shows that the imprint of the nails is clearly evident within the copper at a plane $3 \mathrm{~mm}$ from the impact surface. However, assigning strength to the copper using the Johnson-Cook model [3] dramatically reduced the perturbations in the target as shown by comparing Fig. $2 b$ with Fig. 2a. Note that the identifiers for the simulations in Fig. 2 (ggh5ag and ggh1bg) are superimposed on these and most of the other simulations in the paper. These identifiers have been added to allow the corresponding $\mathrm{CTH}$ simulations to be easily identified.

Figure 3 shows a section through the $y-z$ plane framed just before the leading edge of the wave reached the back of the 16-mm copper target. Although perturbations are just visible in the target, these appear trivial compared with those in the impactor.

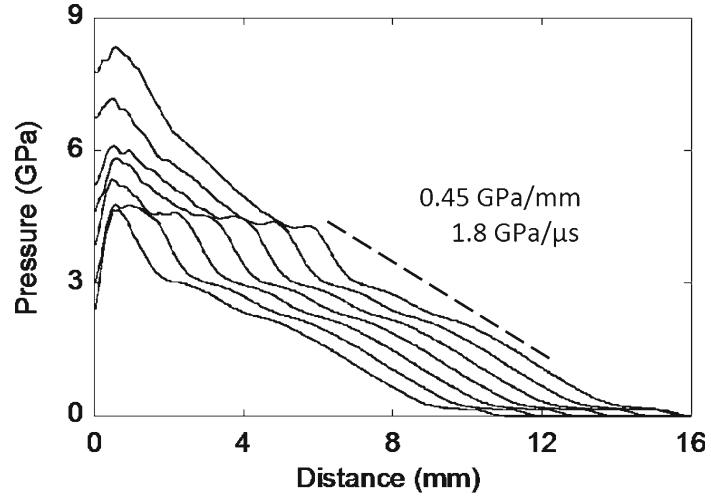

Fig. 4 Wave profiles in a 16-mm copper target impacted at $900 \mathrm{~m} / \mathrm{s}$. The traces are $0.2 \mu \mathrm{s}$ apart. The slope of the dashed line is $\sim 0.45$ $\mathrm{GPa} / \mathrm{mm}$ which, assuming a wave velocity of $\sim 3.94 \mathrm{~mm} / \mu \mathrm{s}$, gives a time gradient of $1.8 \mathrm{GPa} / \mu \mathrm{s}$

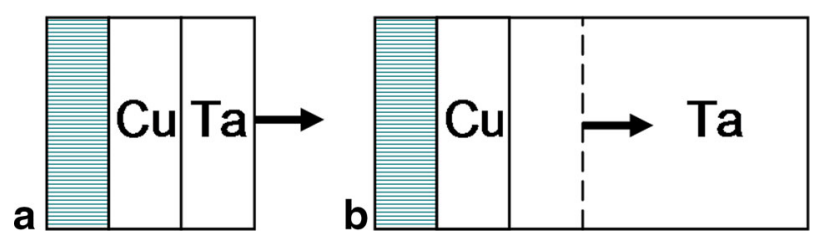

Fig. 5 a and b set-ups for simulations to determine in situ and free surface particle velocities

Figure 4 shows a series of pressure vs distance profiles corresponding to the simulation in Fig. 3. Note that, since the target was impacted on its left face, the waves propagate to the right. As indicated by the dashed line, the pressure in the wave rises by $\sim 0.45 \mathrm{GPa} / \mathrm{mm}$ (equivalent to $\sim 1.8$ $\mathrm{GPa} / \mu \mathrm{s})$.

It was concluded from our study of the impact of the BON flyer on a thick copper target that the method could be used to deliver a ramp wave which was reasonably linear in its rate of rise and furthermore was reasonably smooth on a scale comparable with the spacing of the nails in the pilot design.

In the next phase of the $\mathrm{CTH}$ simulations, computing requirements were much reduced by adopting the "cut down" configuration depicted in Fig. 1b. The simulated region consists of a $2 \mathrm{~mm} \times 2 \mathrm{~mm}$ element with reflective boundaries on the $x-z$ and $y-z$ planes. Comparison between full and cut-down simulations showed insignificant differences, justifying the use of the cut-down configuration.

The next objective of the CTH study was to assess the smoothness of the ramp wave delivered to a possible sample material. In the configuration shown in Fig. 5a, the ramp wave was transmitted via a 6-mm copper smoothing layer to a 6-mm Tantalum target. Again, a picture of the calculated perturbations in the copper/tantalum acceptor is provided by imaging the $x-y$ plane as the wave passes through it. The time sequence in Fig. 6 shows the simulated velocity distribution halfway through the 6-mm copper "buffer" depicted in Fig. 
5a. The times indicated are measured from impact time and the grey scales show calculated velocities in metres per second. Although the "imprint" of the nails is significant within the early part of the wave, the fractional variation in velocities across the $x-y$ plane has almost disappeared in the later part of the wave. The sequence in Fig. 7 shows the predicted velocity at the back surface of the tantalum coupon. Note the very narrow velocity limits in the scale adjacent to the velocity maps. It can be seen that the predicted perturbation at the back surface of the tantalum is $\sim 0.5 \%$ of the mean velocity. It was concluded from these simulations that the wave delivered to, and emerging from, the tantalum sample was acceptably smooth across planes perpendicular to the wave propagation direction. Simulated velocity vs. time profiles corresponding to the configurations depicted in Figs. 5, 6 and 7 are shown in Fig. 8.

Note that the configuration in Fig. 5a is a plausible experimental configuration; the simulation of the surface velocity

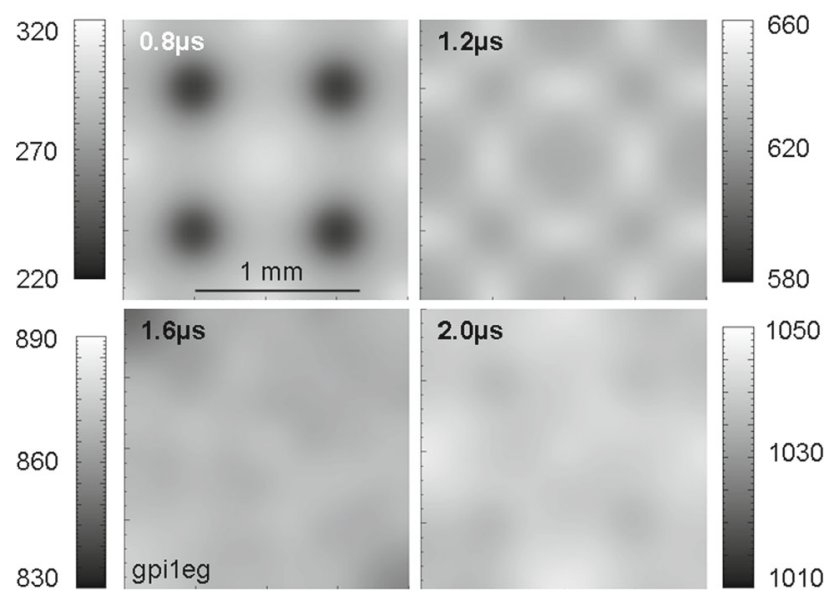

Fig. 6 CTH simulations of the configuration shown in Fig. 5 (a), (with a 3-mm tantalum coupon). Velocity distributions, in $\mathrm{m} / \mathrm{s}$, midway through the 6-mm copper buffer are shown

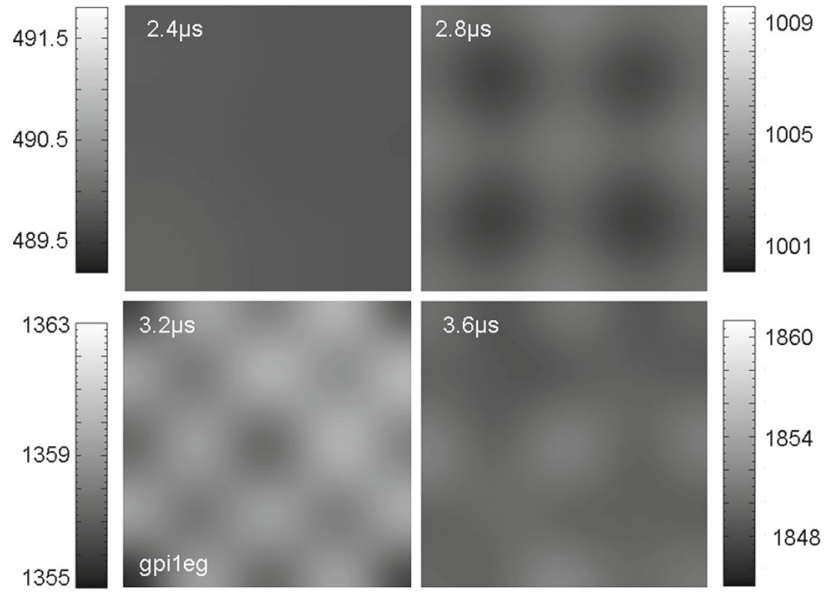

Fig. 7 CTH simulations of the configuration shown in Fig. 5 (b), showing the velocity distributions at the back face of a 3-mm tantalum coupon of the tantalum coupon in Fig. 5a provided an estimate of the wave profile that could be checked against experiment. However, to unfold such an experiment to yield data about the sample material, we need to know what the profile would have been if the profile had not been perturbed by the free surface. In other words, we need the "in situ" wave profile. CTH allows us to compare the simulated in situ velocities with the in situ velocities derived from free surface velocities. Free surface velocities were computed by running the configurations shown in Fig. 5a with coupon thicknesses of 3,5 , and $7 \mathrm{~mm}$. In situ velocities computed at locations 3, 5 and $7 \mathrm{~mm}$ into the thick tantalum sample are shown as solid red lines in Fig. 8. The computed surface velocities, depicted as blue lines in Fig. 8 have been divided by two to allow comparison with the corresponding in situ velocities. It is seen that to a close approximation the in situ velocity is half the free surface velocity, as would be expected from simple hydrodynamic analysis. This approximation will be used later to analyse the experimental results.

Finally, in this section, Fig. 9 shows pressure vs. distance plots for the configuration shown in Fig. 5b. It is seen that the rate of pressure increase in the wave is similar to that observed when the ramp was delivered directly to a thick copper target.

Based on the simulations presented in this section, it was judged that the configuration depicted in Fig. 5b should form the basis of an experimental study.

\section{Experiments}

Two nominally identical samples of the BON flyer were manufactured using the SLM technique. As outlined earlier, each flyer incorporated a protective cylinder of $316 \mathrm{~L}$ stainless

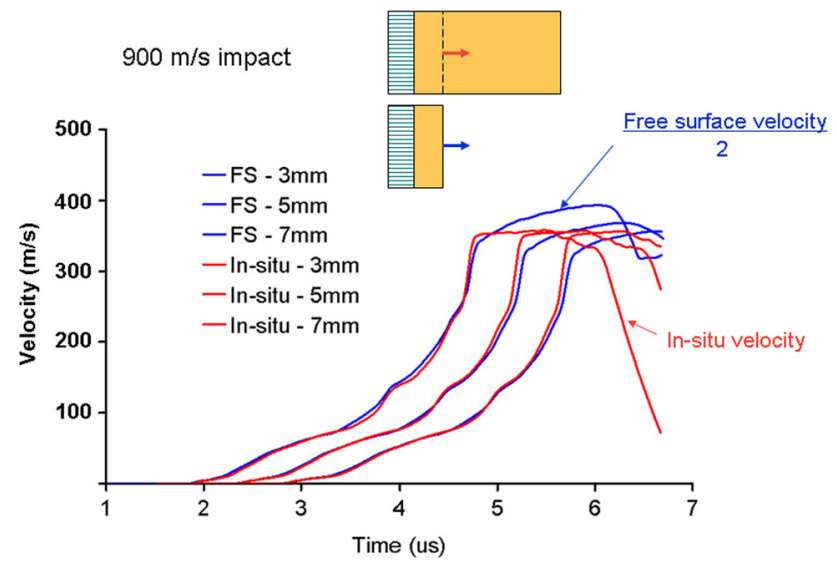

Fig. 8 CTH comparisons of in situ and free surface velocities. It is seen that over most of the profile, the in situ trace estimated by dividing the free surface velocity by two agrees well with the actual simulated in situ velocity profile 


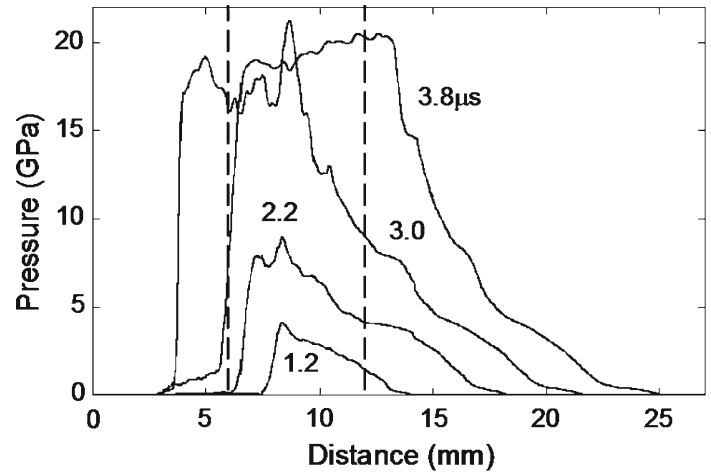

Fig. 9 Pressure plots for $900 \mathrm{~m} / \mathrm{s}$ impact onto a target consisting of 6$\mathrm{mm}$ copper plus $16 \mathrm{~mm}$ of tantalum. As indicated by the dashed lines, the initial positions of the $\mathrm{BON} / \mathrm{Cu}$ and $\mathrm{Cu} / \mathrm{Ta}$ interfaces are at 6 and 12 $\mathrm{mm}$, respectively
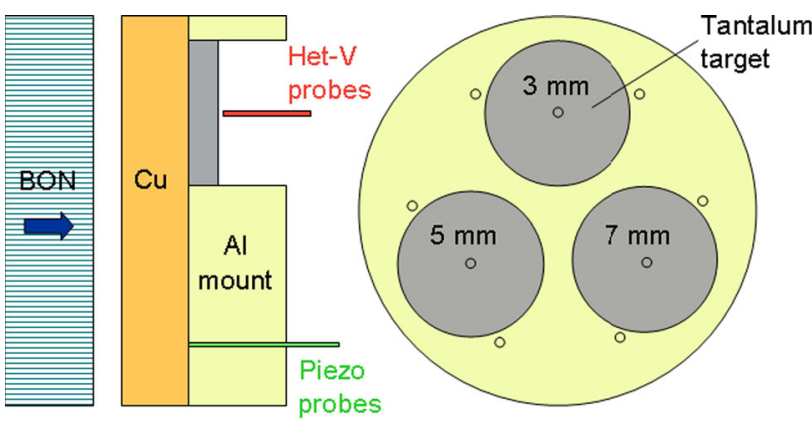

Fig. 10 Experimental set-up

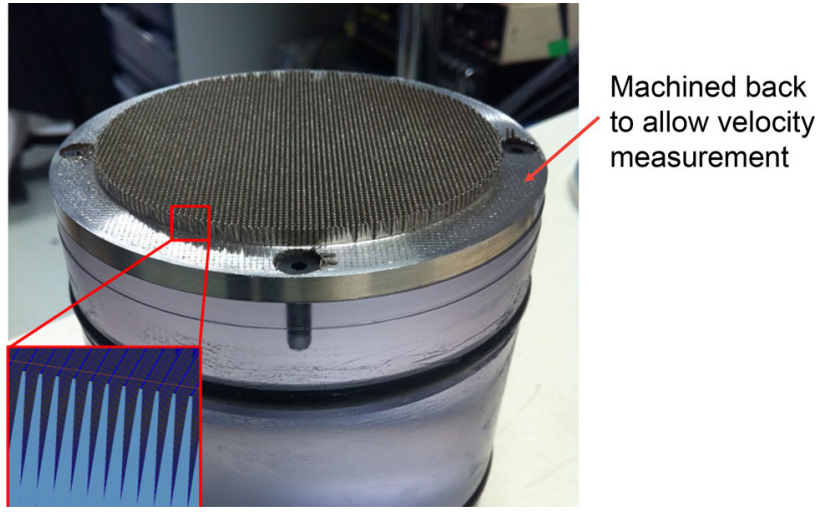

Fig. 11 Photograph of BON flyer

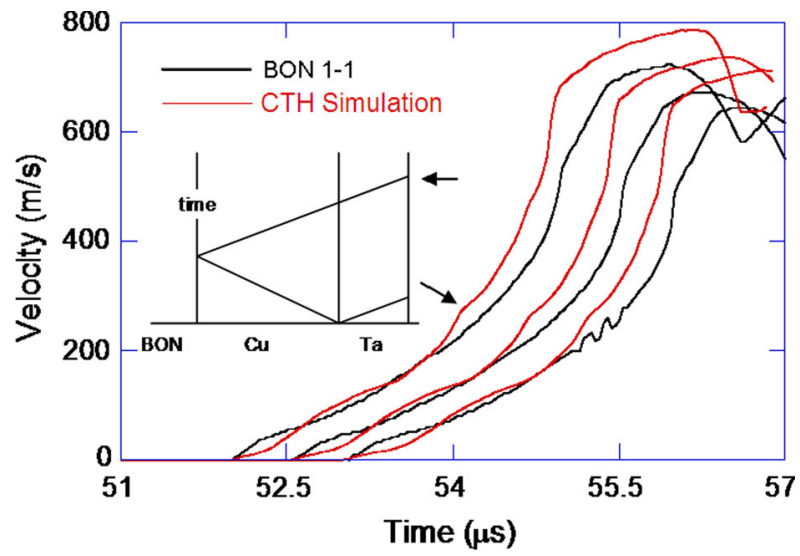

Fig. 12 Results of $900 \mathrm{~m} / \mathrm{s}$ experiment for comparison with the mass corrected CTH simulation. It is believed that the jump in the CTH trace (indicated by the black arrow) is the result of a wave reflected from the BON/copper interface (also indicated by a black arrow)

\section{Results}

The measured surface velocities for the three target thicknesses are shown for the $900 \mathrm{~m} / \mathrm{s}$ experiment (termed BON1-1) as black lines in Fig. 12. The traces were compared with CTH predictions run using the stl file that was used to manufacture the specimens. In these initial simulations, it was found that the simulated velocity profile lay significantly below the experimental observation. We concluded that the reason for the discrepancy was that the mass corresponding to the stl file was, at $299 \mathrm{~g}$, significantly lower than the measured value of $330 \mathrm{~g}$. To allow a meaningful code vs. experiment comparison, a new stl file was created by moving the outer surface of the BON sample outwards until the stl mass matched the measured mass. It was found that an offset of $0.05 \mathrm{~mm}$ was needed to obtain a match. The nail profile used for the comparison shown in Fig. 12 is indicated by the dashed profile in Fig. 1c; it will be referred to in the remainder of this paper as the "adjusted-mass" profile. 


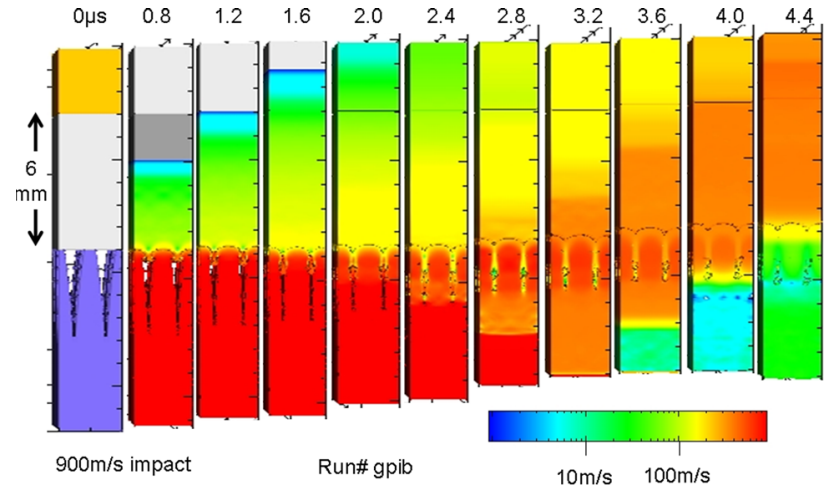

Fig. 13 Velocity plots for a $900 \mathrm{~m} / \mathrm{s}$ impact of a mass corrected BON flyer onto a target consisting of $6 \mathrm{~mm}$ of copper and $3 \mathrm{~mm}$ of tantalum. Note that the zero time frame is a material plot. The first motion of the free surface of the tantalum occurs at $\sim 2 \mu \mathrm{s}$. A wave reflected from the $\mathrm{BON} /$ copper interface arrives $\sim 2 \mu \mathrm{s}$ later (at $\sim 4 \mu \mathrm{s}$ )

Overall, we felt that the simulation using the adjusted mass stl file matched the experiment reasonably well. In particular, it is interesting that the measured traces appear smoother than the CTH traces. The main discrepancy is that in the CTH traces there is a noticeable jump in velocity at $\sim 2 \mu \mathrm{s}$ after wave arrival, which was not observed experimentally. This jump displaces the simulated trace upwards for the remainder of the record. We note in the schematic distance-time plot, inset in Fig. 12, that the timing of the jump suggests that it arises from a reflection at the $\mathrm{BON} /$ copper interface. This hypothesis is further supported by examination of the time sequence shown in Fig. 13. This sequence, which again was run with the adjusted mass stl file, shows particle velocity maps. (Note, however, that the zero time frame, positioned at the extreme left shows a material, rather than a velocity, plot). Examination of the CTH velocity maps, in Fig. 13, suggests that the velocity jump seen in Fig. 12 arises from a reflection at the BON/copper interface. We are unsure why this feature is absent in the experimental traces, but it is possible that the interface presents a more dispersed density profile in the experiment than that computed; this could reduce the sharpness of the reflected wave.

If it is assumed that the free surface velocities are twice the in situ velocities, as suggested by the CTH calculations in Fig. 8, the shock wave conservation relations can be used to generate longitudinal stress vs. specific volume plots or longitudinal stress vs. engineering strain plots corresponding to each of the three pairs of results available from the data. Analysis of the data to extract the material properties requires the Lagrangian sound speed to be calculated by determining the time to reach a given value of particle velocity in targets of different thickness. The equations used to generate the longitudinal stress vs. volume relationships are as follows:

$$
c_{\mathrm{L}}=\left(z_{2}-z_{1}\right) /\left(t_{2}\left(u_{\mathrm{p}}\right)-t_{1}\left(u_{\mathrm{p}}\right)\right)
$$

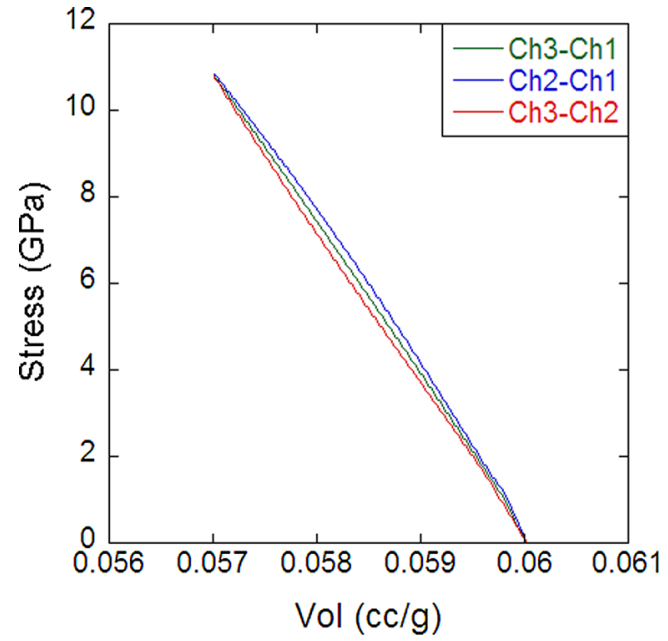

Fig. 14 Stress vs. volume plots for the three pairs of records provided by the $900 \mathrm{~m} / \mathrm{s}$ experiment

$$
\begin{aligned}
\mathrm{d} \sigma & =\rho_{0} c_{\mathrm{L}} d u_{\mathrm{p}} \\
\mathrm{d} V & =-V_{0}\left(d u_{\mathrm{p}} / c_{\mathrm{L}}\right) \\
\mathrm{d} \varepsilon^{\mathrm{E}} & =d u_{\mathrm{p}} / c_{\mathrm{L}}
\end{aligned}
$$

where $c_{\mathrm{L}}$ is Lagrangian longitudinal sound speed, $u_{\mathrm{p}}$ the particle velocity, $\rho_{0}$ the initial density, $z$ the target thickness, $t\left(u_{\mathrm{p}}\right)$ the time at which particle velocity, $u_{\mathrm{p}}$, is reached, $\sigma$ the longitudinal stress, $V$ the volume and $\varepsilon^{\mathrm{E}}$ the engineering strain.

As each experiment recorded data from three targets of different thicknesses, there are three routes to calculate the stress vs. volume relationship depending on which two channels of data are selected. The plots of longitudinal stress vs. specific volume for all three routes are shown in Fig. 14. Note that (4) would allow stress to be plotted against engineering strain if required. It is seen that the three stress vs. volume curves are slightly different from each other suggesting that there are some errors in the method. Possible sources of error include uncertainties over the projectile tilt and the arrival time of the ramp at the front face of the tantalum target. In addition, it is possible that the composition of the flyer varied across its face. For instance, some of the nails, (or groups of nails), could have had a slightly different shape or composition from others. Variations of this type could lead to differences in the waves delivered to the different tantalum samples.

\section{Conclusions}

An stl file was generated for a flyer in which the average density varies linearly over a distance of $5.5 \mathrm{~mm}$. The Sandia National Laboratories code CTH suggested that, at $900 \mathrm{~m} / \mathrm{s}$, this would deliver a pulse with a rise time of $\sim 1.8 \mathrm{GPa} / \mu \mathrm{s}$ to a copper target. Bed-of-nails flyers were manufactured by 
exporting an stl file from a CAD package to a "3D Printer". However, it was found that the mass of the components was greater than the mass corresponding to the stl file. A "corrected" stl file was created by moving the outer profile of the nails outwards. Experiments were conducted in which Het-V was used to measure the free surface velocities of tantalum target discs of thicknesses 3,5 and $7 \mathrm{~mm}$. The measured profiles appeared reasonably smooth and in broad agreement with the simulations. Simulations run using the corrected stl file were compared with the experimental results and a reasonable match was obtained. However, the simulations suggested that the profile would be affected by reflections from the impact interface. Assuming that the surface velocity is twice the in situ velocity allows longitudinal stress vs. specific volume curves (and stress vs. engineering strain) to be derived.

The work reported here showed that there can be significant differences between the ideal design as defined by the stl file and the actual design generated by the SLM process. Clearly, in future work, methods should be sought of determining the actual structure of the samples and, in particular, the uniformity of the structure across the face of the flyer. Possible approaches include radiographic tomography, and sectioning followed by optical examination.

Improvements will be made in the recording of the projectile tilt and arrival time of the ramp wave at the sample. In future experiments, the moment of impact between the flyer and the sample will be recorded by a ring of piezoelectric probes and the arrival of the ramp at the back face of the copper will be recorded using additional Het- $\mathrm{V}$ channels.

Finally, we note that assigning strength to a copper acceptor has the effect of damping the perturbations initially generated in the copper by the tips of the impacting nails. This observation leads us to speculate whether measuring the rate of decay of perturbations in the target could provide a measure of the strength of the target material under dynamic loading. The concept is akin to that pioneered by Sakharov et al. in [8].
Acknowledgments The experiments were performed using the 100$\mathrm{mm}$ gas gun at the Institute of Shock Physics, Imperial College London. We thank Mark Swan and Nick Gibbs, AWE for helping to prepare the samples and Steven Johnson, ISP for help with the experiments. AWE and Imperial College London are acknowledged for their continued support of the Institute of Shock Physics.

Open Access This article is distributed under the terms of the Creative Commons Attribution 4.0 International License (http://creativecomm ons.org/licenses/by/4.0/), which permits unrestricted use, distribution, and reproduction in any medium, provided you give appropriate credit to the original author(s) and the source, provide a link to the Creative Commons license, and indicate if changes were made.

\section{References}

1. Davis, J.P.: Experimental measurement of the principal isentrope for aluminium 6061-T6 to 240GPa. J. Appl. Phys. 99, 103512 (2006). doi: $10.1063 / 1.2196110$

2. Asay, J. R., Vogler, T. J., Ao, T., Ding, J. L.: Dynamic yielding of single crystal Ta at strain rates of similar to $5 \times 10^{5} / \mathrm{s}$. J. Appl. Phys. 109073507 (2011). doi:10.1063/1.3562178

3. McGlaun, J.M., Thompson, S.L., Elrick, M.G.: CTH: a threedimensional shock wave physics code. Int. J. Impact. Eng. 10, 351360 (1990). doi:10.1016/0734-743X(90)90071-3

4. Steinberg, D.J., Cochran, S.G., Guinan, M.W.: A constitutive model for metals applicable at high strain rates. J. Appl. Phys. 51, 14981504 (1980). doi:10.1063/1.327799

5. Johnson, G.R., Cook, W.H.: A constitutive model and data for metals subjected to large strains, high strain rates and high temperatures. In: Procedings of the 7th International Symposium Ballistics, Netherlands, 541-547 (1983)

6. Winter, R.E., Cotton, M., Harris, E.J., Maw, J.R., Chapman, D.J., Eakins, D.E., McShane, G.: Plate-impact loading of cellular structures formed by selective laser melting. Model. Simul. Mater. Sci. Eng. 22, 025021 (2014). doi:10.1088/0965-0393/22/2/025021

7. Strand, O.T., Goosman, D.R., Martinez, C., Whitworth, T.L., Kuhlow, W.W.: Compact system for high-speed velocimetry using heterodyne techniques. Rev. Sci. Instrum. 77083108 (2006). doi:10. $1063 / 1.2336749$

8. Sakharov, A.D., Oleinik, A.G., Mineev, V.N., Zaidel, R.M.: Experimental investigation of stability of shock waves and mechanical properties of matter at high pressures and high temperatures. Doklady Akademii Nauk SSSR 159, 1019-1022 (1964) 